\title{
Germinação de sementes de cenoura osmoticamente condicionadas e peletizadas com diversos ingredientes
}

\author{
Warley M Nascimento ${ }^{1}$; João Bosco C. Silva ${ }^{1}$; Paulo EC Santos ${ }^{2}$; Ricardo Carmona ${ }^{3}$ \\ ${ }^{1}$ Embrapa Hortaliças, C. Postal 218, 70359-970 Brasília-DF; ${ }^{2}$ professor da FTB; ${ }^{3}$ UnB, Campus Universitário Darci Ribeiro, Asa Norte, \\ 70910-900 Brasília-DF; wmn@cnph.embrapa.br
}

\begin{abstract}
RESUMO
A semente de cenoura caracteriza-se por ser relativamente pequena, o que dificulta o processo de distribuição, tornando a mecanização por semeadeiras convencionais uma prática difícil. A técnica da peletização tem sido utilizada visando a melhoria da distribuição de sementes e o estabelecimento de plântulas no campo. As sementes peletizadas geralmente apresentam menor velocidade de germinação quando comparadas com as sementes nuas do mesmo lote. $\mathrm{O}$ condicionamento osmótico tem sido utilizado para aumentar a velocidade de germinação de sementes. Neste estudo foram avaliados materiais cimentantes para a peletização de sementes e verificadas as relações existentes entre o condicionamento osmótico e a germinação das sementes peletizadas de cenoura cv. Alvorada. A peletização foi feita utilizando-se, como material de enchimento, a mistura de microcelulose e areia fina em volumes iguais e, para enchimento foram avaliados cinco materiais cimentantes: bentonita, methocel, opadry, rhoximat e goma arábica. Sementes peletizadas receberam ou não o acabamento com iriodin. Uma testemunha (sementes nuas) foi incluída no ensaio. Em outro estudo, sementes foram osmoticamente condicionadas em solução aerada de polietileno glicol e peletizadas imediatamente após o condicionamento osmótico ou após a secagem. Os produtos rhoximat e opadry apresentaram as melhores características como material cimentante, formando uma fina camada de revestimento, que resultou na formação de péletes com superfície lisa, uniforme e ótima aparência externa. Em geral, péletes apresentaram uma ligeira redução na porcentagem e na velocidade de germinação, em comparação às sementes não peletizadas, com diferenças maiores para os péletes que receberam o acabamento com iriodin. $\mathrm{O}$ condicionamento osmótico aumentou a velocidade de germinação das sementes peletizadas de cenoura.
\end{abstract}

Palavras-chave: Daucus carota, plantabilidade, estabelecimento de plantulas.

\section{ABSTRACT \\ Germination of carrot seed primed and pelleted with several ingredients}

Carrot seeds are small and the distribution in the field by seeders is generally very difficult. Seed pelleting has been used to improve sowing and consequently the stand establishment. Pelleted seeds generally present lower germination rate than raw seeds from the same seed lot. Seed priming has been used to increase germination rate of several species. Various binder materials for seed pelleting were evaluated and the relation between the osmoconditioning and germination of pellet seeds verified, using 'Alvorada' carrot cultivar. The pellet was obtained through a mixture of equal parts of microcelulose and sand as filling materials; five binder materials were also evaluated: bentonite, methocel, opadry, rhoximat and arabic gum. Part of the pelleted seeds received an external coat treatment (iriodin). Raw seeds were included as control. In another study, seeds were primed in an aerated solution of polyethylene glycol, dried and then pelleted. Rhoximat and opadry showed better characteristics as binder materials presenting pellets with smooth surface, uniform and excellent external appearance. In general, seed pellets showed a slight decrease of the germination rate and total germination compared to raw seeds, with greather differences for pellets finished with iriodin. Priming enhanced the germination rate of pelleted carrot seeds.

Keywords: Daucus carota, plantability, stand establishment.

\section{(Recebido para publicação em 29 de novembro de 2006; aceito em 17 de fevereiro de 2009) (Received in November 29, 2006; accepted in February 17, 2009)}

\begin{abstract}
A semeadura direta é o processo requerido por hortaliças que não toleram o transplante e/ou sofrem deformações das suas partes comerciais, tais como a cenoura. Nesta cultura, a semeadura pode ser realizada manual ou mecanicamente. Em ambos os casos temse, normalmente, uma população de plantas bem superior àquela ideal. $\mathrm{O}$ gasto excessivo de sementes é normalmente considerado pelo produtor como garantia de plântulas suficientes para superar problemas relacionados com a qualidade fisiológica das sementes e/ou
\end{abstract}

adversidades ambientais. Entretanto, a prática do desbaste eleva o custo de produção, que no caso da cenoura pode corresponder a cerca de $12 \%$ (Vieira et al., 1997).

A semente de cenoura caracteriza-se por ser relativamente pequena (600 a 850 sementes por grama), possuir pouca reserva nutritiva e ser dotada de epitélio rugoso, o que dificulta a distribuição, tornando a mecanização por semeadeiras convencionais não muito fácil. Além disso, as sementes de cenoura apresentam germinação desuniforme devido à pre- sença de inibidores no epitélio e alta sensibilidade a extremos de umidade e temperatura durante a fase de germinação (Currah et al., 1974).

Vários tratamentos de sementes têm sido propostos com a finalidade de minimizar tais problemas, dentre eles, a peletização de sementes, que tem demonstrado ser uma técnica bastante promissora. A peletização consiste no revestimento das sementes com um material seco, inerte, de granulometria fina e um material cimentante (adesivo). Este tratamento permite dar à semente uma 
forma arredondada, aumentando o seu tamanho, facilitando assim a sua distribuição, seja ela manual ou mecânica. $\mathrm{Na}$ cultura da cenoura, por exemplo, a utilização de sementes peletizadas reduz os gastos excessivos de sementes e a prática de desbaste. Embora a técnica de peletização tenha sido desenvolvida há vários anos, as informações referentes à confecção dos péletes são pouco difundidas, uma vez que esta técnica permanece inacessível junto às companhias de sementes e/ou empresas processadoras dos péletes. Os materiais empregados na peletização, incluindo aqueles de cobertura, adesivos e de acabamento, influenciam na rigidez do pélete, na absorção de água e na troca gasosa entre a semente e o ambiente externo ao pélete e todos estes aspectos afetam diretamente a germinação das sementes (Silva, 1997; Silva \& Nakagawa, 1998a).

Os produtos a serem utilizados como cimentantes devem ter afinidade com os demais ingredientes, ser prontamente solúvel em água, atuar em baixa concentração, se tornar seco e não pegajoso quando desidratado, formar solução de baixa viscosidade ao ser reidratado, não ser higroscópico, corrosivo e nem tóxico, ser estéril e não constituir meio de crescimento de microrganismos.

É necessário que os materiais adesivos sejam realmente solúveis em água e não que apenas tenham sua consistência reduzida quando umedecidos. A diferença pressuposta é que, havendo a solubilização do produto, ocorra o seu carreamento para fora do pélete e consequentemente diluição do seu efeito adesivo (Silva, 1997). Os adesivos são, geralmente, produtos viscosos, e a viscosidade da solução ou da suspensão depende da concentração. A afinidade entre o cimentante e os demais ingredientes, ou o equivalente ao poder de fixação do produto é importante, para que assim se utilize o menor volume de solução de cimentantes no pélete.

A integridade física dos péletes é outra característica muito importante, pois não devem desmanchar ou quebrar durante os processos de classificação, transporte, manuseio e semeadura mecanizada. Para evitar a sua desintegração, são utilizados os cimentantes (ade- sivos), que devem ter como principais propriedades: não serem fitotóxicos; terem afinidade com os demais ingredientes; serem solúveis em água; atuarem em baixa concentração; e devem formar solução de baixa viscosidade ao serem reidratados (Silva et al., 1998b).

Apesar dos benefícios da peletização, o processo de revestimento de sementes pode afetar a germinação devido à barreira imposta pelo material, dificultando a emissão da raiz primária, a troca de gases e a sua difusão para o ambiente externo ao pélete (Tonkin, 1979; Silva, 1997). Por exemplo, sementes peletizadas de alface não submetidas previamente ao processo de condicionamento osmótico, geralmente apresentam menor velocidade de germinação quando comparadas com as não peletizadas (Silva et al., 2002). Outros estudos têm demonstrado que sementes nuas de várias espécies germinam mais rápido do que as sementes peletizadas (Sachs et al.,1981; Tonkin, 1984).

O condicionamento osmótico pode melhorar a uniformidade e a velocidade de germinação de sementes de cenoura (Dearman et al., 1987; Peluzio et al., 1999). Este tratamento consiste da hidratação controlada das sementes, suficiente para promover atividades prémetabólicas, sem contudo permitir a emissão da raiz primária. As sementes são imersas em solução osmótica por períodos de tempo e temperaturas determinados. O potencial osmótico da solução regula a hidratação das sementes, permitindo a ativação de processos metabólicos nas fases iniciais da germinação, evitando a emergência da raiz primária (Heydecker, 1973).

Os objetivos deste estudo foram avaliar materiais cimentantes para a peletização de sementes de cenoura e verificar as relações existentes entre o condicionamento osmótico e a germinação das sementes peletizadas.

\section{MATERIAL E MÉTODOS}

\section{Peletização de sementes - Sementes} de cenoura cv. Alvorada foram peletizadas na Embrapa Hortaliças, utilizando-se uma betoneira adaptada com rotação de 25 a 30 rpm, onde as sementes sofreram inúmeras rotações em tor- no de seu próprio eixo, com tendência a rolarem umas sobre as outras. Os materiais de enchimento e os agentes cimentantes foram aplicados aos poucos sobre a massa de sementes em movimento, com auxílio de uma peneira de malha fina, alternando com a pulverização cuidadosa de água, de forma a umidificar a massa sem formação de pasta.

Como material de enchimento, utilizou-se a mistura homogeneizada de areia fina (100 micras) e microcelulose em volumes iguais e em quantidade correspondente a três vezes o peso das sementes. Quatro materiais cimentantes foram testados: bentonita $\AA$, opadry $\AA$, rhoximat $\AA$ e goma arábica, todos na proporção de $5 \%$ em relação ao peso dos materiais de enchimento. No final do processo de peletização, as sementes receberam ou não acabamento com iriodin $®$ a $5 \%$, perfazendo assim um total de oito tratamentos. O produto methocel® foi também avaliado como cimentante, mas os péletes foram descartados.

Imediatamente após a confecção, os péletes foram colocados para secar a $25^{\circ} \mathrm{C}$ durante 24 horas e passados em três peneiras de furos redondos CAT 106 (Modelos 2.8; 2.6 e 1.8), a fim de eliminar péletes muito grandes ou muito pequenos, resultando assim em um material mais homogêneo. Sementes nuas, sem sofrerem o processo de peletização, foram incluídas como testemunha. Quatro repetições de 50 sementes por tratamento, em um delineamento inteiramente casualizado, foram colocadas para germinar em gerbox, contendo duas folhas de papel germibox previamente umedecidas com $5 \mathrm{~mL}$ de água destilada e incubadas a $20-30^{\circ} \mathrm{C}$, conforme as Regras para Análise de Sementes (Brasil, 1992). O substrato foi mantido suficientemente úmido durante todo o teste a fim de dar às sementes a quantidade de água necessária para a germinação. A emissão da radícula foi avaliada diariamente, sendo as contagens de germinação realizadas aos 7 e 14 dias. Os dados obtidos neste estudo foram submetidos à análise de variância, comparando as médias dos tratamentos por meio do teste Tukey a 5\% de probabilidade.

Condicionamento osmótico de sementes - Sementes de cenoura cv. Alvorada foram osmoticamente condiciona- 
Tabela 1. Germinação e velocidade de germinação a $20-30^{\circ} \mathrm{C}$ de péletes de sementes de cenoura cv. Alvorada, confeccionados com materiais cimentantes, com e sem acabamento com Iriodin (germination and velocity of germination at $20-30^{\circ} \mathrm{C}$ of pelleted carrot Alvorada seeds, using binder material and Iriodin or not). Brasília, Embrapa Hortaliças, 2001.

\begin{tabular}{|c|c|c|c|c|c|c|}
\hline \multirow{4}{*}{$\begin{array}{l}\text { Tratamentos } \\
\text { (materiais } \\
\text { cimentantes) }\end{array}$} & \multicolumn{4}{|c|}{ Germinação(\%) } & \multirow{2}{*}{\multicolumn{2}{|c|}{$\begin{array}{c}\text { Velocidade de } \\
\text { germinação (IVG) }\end{array}$}} \\
\hline & \multirow{2}{*}{\multicolumn{2}{|c|}{$\frac{7 \text { dias }}{\text { Acabamento }}$}} & \multirow{2}{*}{\multicolumn{2}{|c|}{$\frac{14 \text { dias }}{\text { Acabamento }}$}} & & \\
\hline & & & & & \multicolumn{2}{|c|}{ Acabamento } \\
\hline & Sem & Com & Sem & Com & Sem & Com \\
\hline Testemunha & $73 \quad a$ & $73 \quad a^{1}$ & 85 & 85 & $3,57 \quad b$ & 3,57 \\
\hline Bentonita & $65 \mathrm{Aa}$ & $49 \mathrm{~A} \quad \mathrm{~b}$ & $79 \mathrm{~A} a b$ & $71 \mathrm{Ab}$ & $4,47 \mathrm{Aa}$ & $3,99 \mathrm{~A} \mathrm{a}$ \\
\hline Opadry & $64 \mathrm{~A} \mathrm{a}$ & $57 \mathrm{~A} a b$ & $78 \mathrm{~A} \quad \mathrm{~b}$ & $73 \mathrm{Ab}$ & $4,29 \mathrm{~A} \mathrm{a}$ & $3,81 \mathrm{~B} \mathrm{ab}$ \\
\hline Goma arábica & $61 \mathrm{Aa}$ & $54 \mathrm{~A} a b$ & $76 \mathrm{~A} \quad \mathrm{~b}$ & $70 \mathrm{~B} \mathrm{~b}$ & $4,51 \mathrm{~A} \mathrm{a}$ & $4,16 \mathrm{Aa}$ \\
\hline Rhoximat & $59 \mathrm{Aa}$ & $59 \mathrm{~A} a b$ & $78 \mathrm{~A} \mathrm{~b}$ & $78 \mathrm{~A} a b$ & $4,48 \mathrm{Aa}$ & $4,23 \mathrm{~A} \mathrm{a}$ \\
\hline Média & $64,4 \mathrm{~A}$ & $58,4 \mathrm{~A}$ & $79,2 \mathrm{~A}$ & $75,4 \mathrm{~B}$ & $4,27 \mathrm{~A}$ & $3,95 \mathrm{~B}$ \\
\hline CV (\%) & 28,21 & 28,90 & 7,39 & 9,89 & 10,58 & 9,73 \\
\hline
\end{tabular}

${ }^{1}$ Médias seguidas de mesma letra maiúsculas nas linhas e minúsculas nas colunas, não diferem entre si pelo teste Tukey a $5 \%$ de probabilidade. ${ }^{2}$ Índice de velocidade de germinação (IVG) obtido com a fórmula proposta por Maguire (1962) ( ${ }^{1}$ means followed by the same small letter in the column and capital letter in the rows did not differ from each other through the Tukey test, 5\%; ${ }^{2}$ index of germination rate (IVG) obtained through Maguire's formula (1962)).

das em solução aerada de polietileno glicol $(30 \%)$ por 48 horas a $15^{\circ} \mathrm{C}$, sob luz. A aeração foi provida por uma bomba de aquário. Em seguida, parte das sementes foi lavada em água corrente, secada superficialmente por alguns minutos e peletizada. Outra parte foi secada a $15^{\circ} \mathrm{C}$ durante 48 horas e também peletizada. Utilizou-se, como enchimento, uma mistura de areia e de microcelulose em partes iguais e o produto rhoximat como cimentante. Os dados obtidos neste estudo foram submetidos à análise de variância, comparando as médias dos tratamentos através do teste Tukey a $5 \%$ de probabilidade.

\section{RESULTADOS E DISCUSSÃO}

Peletização de sementes - Dentre os produtos testados, os melhores resultados em termos visuais foram obtidos com os produtos rhoximat e opadry. $\mathrm{O}$ opadry é um material de granulometria muito fina, formando uma fina camada de revestimento, que resulta na formação de péletes com ótima aparência externa, sendo na maioria das vezes, mais leves quando comparados aos demais. Assim, este material apresenta um potencial para a peliculização de sementes. Com o produto rhoximat formaramse péletes de boa aparência, com formato mais cilíndrico, mais pesados do que aqueles confeccionados com o produto opadry e com uma camada de revesti- mento mais rígida e espessa.

Os péletes confeccionados com methocel foram descartados em virtude da dificuldade de confecção dos mesmos, formando uma massa pastosa. Este produto, quando em contato com água forma suspensões gelatinosas que, possivelmente, dificultam a drenagem do excesso de água, podendo interferir no processo de troca gasosa entre a semente e o ambiente externo ao pélete. O methocel é utilizado na indústria farmacêutica para a confecção de comprimidos de solubilização rápida. Pode ser que, com ajuste de dosagem e do modo de usar, este produto possa ser útil no processo de peletização de sementes.

Utilizando-se a bentonita, formaramse péletes pouco resistentes que se quebravam e desmanchavam com facilidade, exigindo um maior cuidado no manuseio, o que dificulta sua utilização em maior escala. A bentonita é composta, basicamente, por argila do tipo $2: 1$, muito expansiva, muito plástica e pegajosa, apresentando uma drenagem muito deficiente.

Os péletes confeccionados com goma arábica, embora tenham germinado satisfatoriamente (Tabela 1), favoreceram a proliferação de fungos. Na maioria das vezes, estes produtos adesivos acabam servindo de substrato para proliferação de microrganismos, aumentando a ocorrência de doenças na fase de germinação da semente (Kanashiro et al., 1978).
As sementes peletizadas apresentaram pouca diferença entre si quanto à germinação final; entretanto, existiu uma diferença significativa entre a maioria dos tratamentos de peletização e a testemunha, tanto naqueles que receberam quanto nos que não receberam o acabamento com iriodin (Tabela 1). A velocidade de germinação também foi afetada pelo processo de peletização.

Na primeira contagem de germinação, feita aos 7 dias, observou-se menores diferenças significativas entre as sementes peletizadas e as sementes nuas, mas isso foi devido à desuniformidade na germinação, o que é percebido pelos altos valores dos coeficientes de variação (cerca de 28\%). Na avaliação final, feita aos 14 dias, obteve-se maior uniformidade dos valores e as diferenças, mesmo que reduzidas, foram estatisticamente significativas.

A maioria dos materiais utilizados como adesivos tem a característica de se solidificarem ou, pelo menos, aumentaram sua consistência quando desidratados, podendo formar uma barreira física para a germinação e o crescimento da plântula. Tonkin (1979) ressalta a importância do tipo de material utilizado no recobrimento, especificando que este não deve se desintegrar durante a embebição, formando uma "papa" (massa sem resistência), o que limitaria a passagem de oxigênio e, em alguns casos, da água para o embrião. Trabalhos realizados com sementes de cenoura por Silva (1997) sugeriram que a camada de revestimento atua como barreira para a difusão, alterando a troca gasosa entre a semente e o ambiente externo ao pélete.

Quanto ao acabamento, verificou-se o efeito de interação, sendo que a maior diferença de germinação foi observada para as sementes peletizadas com goma arábica. Péletes sem acabamento alcançaram, em média, 79\% de germinação aos 14 dias, enquanto péletes com acabamento, $76 \%$ e as sementes nuas, $85 \%$. Embora tenha havido algumas combinações de material cimentante e o acabamento em que o efeito de acabamento não tenha sido estatisticamente significativo, o acabamento sempre reduziu a germinação aos 14 dias.

Ao analisarem os índices referentes à velocidade de germinação (IVG), tem- 
se praticamente as mesmas conclusões, embora as interações tenham ocorrido para combinações diferentes de material cimentante e acabamento. As sementes nuas apresentaram menor IVG, o que corresponde ao menor tempo para a germinação.

Condicionamento osmótico de sementes - As sementes peletizadas, tratadas ou não com condicionamento osmótico apresentaram porcentagem de germinação semelhante ao das sementes nuas, ou seja, o condicionamento osmótico e os processos de secagem utilizados após o condicionamento osmótico não influenciaram na porcentagem final de germinação (Tabela 2).

Entretanto, o condicionamento osmótico favoreceu a velocidade da germinação, sendo que as sementes não condicionadas e peletizadas apresentaram maiores índices de velocidade de germinação (IVG=3,02), ou seja, demoraram mais para germinar.

Em outros trabalhos com cenoura, Dearman et al. (1987) verificaram que o condicionamento osmótico permitiu a redução no tempo de germinação e posterior emergência de plântulas em cenoura, enquanto Sampaio \& Sampaio (1998) verificaram uma melhor performance das sementes nuas de cenoura condicionadas, sendo que a velocidade, a uniformidade e a germinação final apresentaram um aumento significativo. Vale salientar que, no presente trabalho, as sementes de cenoura foram submetidas às condições ótimas de germinação, isto é, temperaturas alternadas de $20-30^{\circ} \mathrm{C}$. Em geral, os maiores benefícios do condicionamento osmótico têm sido observados sob condições edafo-climáticas adversas (Nascimento, 1998). Em cenoura, por exemplo, tem-se observado um melhor desempenho das sementes condicionadas em condições de temperaturas altas (Cantliffe \& Elballa, 1994; Pereira \& Nascimento, 2002; Nascimento \& Pereira, 2007). Em alface, Valdes et al. (1985) verificaram melhor desempenho, em condições de campo, nas sementes peletizadas que foram submetidas ao condicionamento osmótico, tanto comparadas às sementes nuas, não submetidas ao condicionamento osmótico quanto às sementes peletizadas sem condicionamento osmótico. Estes auto-

Tabela 2. Germinação e velocidade de germinação de sementes nuas e peletizadas de cenoura 'Alvorada', submetidas ou não ao condicionamento osmótico (germination and germination rate os carrot 'Alvorada' seeds, submitted or not to osmotic conditioning). Brasília, Embrapa Hortaliças, 2001.

\begin{tabular}{lccc}
\hline \multirow{2}{*}{ Tratamentos $^{1}$} & \multicolumn{2}{c}{ Germinação (\%) } & $\begin{array}{c}\text { Velocidade de } \\
\text { germinação }\end{array}$ \\
\cline { 2 - 3 } & $\mathbf{7}$ dias & 14 dias & $2,59 \mathrm{ab}$ \\
Semente nua & $77 \mathrm{a}^{\mathbf{2}}$ & $83 \mathrm{a}$ & $2,40 \mathrm{a}$ \\
Semente nua + CO + SS & $83 \mathrm{a}$ & $83 \mathrm{a}$ & $2,44 \mathrm{a}$ \\
Semente nua + CO + SE & $78 \mathrm{a}$ & $78 \mathrm{a}$ & $3,02 \mathrm{~b}$ \\
Semente peletizada ${ }^{4}$ & $76 \mathrm{a}$ & $78 \mathrm{a}$ & $2,64 \mathrm{ab}$ \\
Semente + CO + SS + peletização & $80 \mathrm{a}$ & $81 \mathrm{a}$ & $2,84 \mathrm{ab}$ \\
Semente + CO + SE + peletização & $78 \mathrm{a}$ & $79 \mathrm{a}$ & 11,53
\end{tabular}

${ }^{1} \mathrm{CO}=$ condicionamento osmótico; $\mathrm{SS}=$ secagem superficial; $\mathrm{SE}=$ secagem a $15^{\circ} \mathrm{C}$ durante 48 horas; ${ }^{2}$ Médias seguidas de mesma letra na coluna não diferem entre si pelo teste Tukey a 5 $\%$ de probabilidade; ${ }^{3}$ Índice de velocidade de germinação (IVG) obtido com a fórmula proposta por Maguire (1962); ${ }^{4}$ A peletização foi feita utilizando a mistura de areia e microcelulose com material de enchimento e com rhoximat como cimentante $\left({ }^{1} \mathrm{CO}=\right.$ osmotic conditioning; $\mathrm{SS}=$ superficial drying; $\mathrm{SE}=$ drying under $15^{\circ} \mathrm{C}$ during 48 hours; ${ }^{2}$ means followed by the same letter did not differ from each other through the Tukey test, $5 \% ;^{3}$ germination rate (IVG) obtained through Maguire's formula (1962); ${ }^{4}$ pelleting of the seeds was done using a mixture of sand and microcelulose as filling materials and rhoximat as binder material).

res observaram que a emergência total das sementes nuas após seis dias de semeadura em campo foi de 18 a $21 \%$, enquanto sementes osmocondicionadas e peletizadas atingiram, para as mesmas condições, valores de 46 a $69 \%$ de germinação, respectivamente. No mesmo estudo foi verificado que a uniformidade e a emergência também foram maiores para sementes que foram submetidas aos processos de condicionamento osmótico seguido de recobrimento, com 91\% de emergência final ocorrendo até o terceiro dia, enquanto para as sementes nuas, apenas $70 \%$ das sementes germinaram. Assim, o processo de pré-tratamento das sementes utilizando o condicionamento osmótico para posterior peletização, pode ser um método bastante eficiente na melhoria da germinação de sementes de cenoura sob condições de campo.

Fica evidente que o material utilizado no processo de revestimento configura, realmente, uma barreira física a ser vencida pela semente e que, possivelmente, seja esta a razão do atraso na velocidade de germinação na maioria dos tratamentos. Porém, os resultados obtidos sugerem que alguns materiais, provavelmente permitem que haja a difusão de gases e água entre a semente e o ambiente externo. Os resultados mostram que o estande final de plântulas obtidas pelos péletes foi próximo ao das sementes nuas. Estudos nesse sentido devem ser enfatizados visando, simultaneamente, alcançar formulações mais adequadas à peletização e minimizar os efeitos deletérios deste processo.

Concluiu-se que os produtos rhoximat e opadry apresentaram as melhores características como material cimentante para a peletização de sementes de cenoura e que as sementes peletizadas e osmoticamente condicionadas apresentaram germinação semelhante à das sementes não peletizadas, mas com maior velocidade de germinação.

\section{REFERÊNCIAS}

BRASIL. 1992. Ministério da Agricultura e Reforma Agrária. Regras para análise de sementes. Brasília, Secretaria Nacional de Defesa Agropecuária. 365p.

CANTLIFFE DJ; ELBALLA M. 1994. Improved germination of carrot at stressful high temperature by seed priming. Proceedings of the Florida State Horticultural Society 107: 121-128.

CURRAH JE; GRAY D; THOMAS TH. 1974. The rate of sowing on germination vegetable seeds using a fluid-drill. Annals of Applied Biology 34: 311.

DEARMAN J; DREW RLK; BROCKLEHURST PA. 1987. Effect of osmotic priming, rinsing and storage on the germination and emergence of carrot seed. Annals of Applied Biology 111: 723727. 
HEYDECKER W; HIGGIS J; GULLIVER RL. 1973. Accelerated germination by osmotic treatment. Nature 246: 42-44.

KANASHIRO M; KAGEYAMA PY; MÁRQUEZ FCM. 1978. Peletização de sementes de Eucalyptus. Instituto de Pesquisa de Estudos Florestais 17: 67-73.

MAGUIRE JD 1962. Speed of germinationaid in selection and evaluation for seedling emergence and vigor. Crop Science 2: 176177.

NASCIMENTO WM 1998. Condicionamento osmótico de sementes de hortaliças: potencialidades e implicações. Horticultura Brasileira 16: 106-109.

NASCIMENTO WM; PEREIRA RS. 2007. Preventing thermo-inhibition in carrot by seed priming. Seed Science and Technology 35: 503-506.

PELUZIO LE; SILVA RF; REIS MS; CECON PR; DIAS DCFS; PELUZIO JBE. 1999. Efeito do condicionamento osmótico na embebição e na germinação de sementes de cenoura (Daucus carota L.). Revista Brasileira de Sementes 21: 161-169.
PEREIRA RS; NASCIMENTO WM. 2002. Utilização do condicionamento osmótico de sementes de cenoura visando a germinação em condições de temperaturas altas. Horticultura Brasileira 20, Suplemento CD-ROM.

SACHS M; CANTLIFFE DJ; NELL TA. 1981. Germination of clay-coated sweet pepper seeds. Journal of the American Society for Horticultural Science 106: 385-389.

SAMPAIO GT; SAMPAIO NV. 1998. Viabilidade, vigor e armazenamento de sementes de cenoura submetidas ao précondicionamento osmótico. Revista Científica Rural 3: 38-45.

SILVA JBC. 1997. Avaliação de métodos e materiais para a peletização de sementes. Botucatu: UNESP. 127p (Tese doutorado).

SILVA JBC; NAKAGAWA J. 1998a. Confecção e avaliação de péletes de sementes de alface. Horticultura Brasileira 16: 151-158.

SILVA JBC; NAKAGAWA J. 1998b. Metodologia para a avaliação de materiais cimentantes para peletização de sementes. Horticultura Brasileira 16: 31-37.
SILVA JBC; SANTOS PEC; NASCIMENTO WM. 2002. Desempenho de sementes peletizadas de alface em função do material cimentante e da temperatura de secagem dos péletes. Horticultura Brasileira 20: 67-70.

TONKIN JHB. 1979. Pelleting and other presowing treatments. Advances Seed Technology 4: 84-105.

TONKIN JHB. 1984. Pelleting and other presowing treatments. In: THOMPSON JR (Ed.) Advances in research and technology of seeds, part 9. Wageningen: ISTA, p. 9597.

VALDES VM; BRADFORD KJ; MAYBERRY KS. 1985. Alleviation of termodormancy in coated lettuce seeds by seed priming. Hortscience 20: 1112-1114.

VIEIRA JV; PESSOA HBSV; MAKISHIMA N; FONTES RR; MESQUITA FILHO MV; SOUZA AF; MAROUELLI WA; PEREIRA W; FRANCA FH; LOPES CA; REIFSCHNEIDER FJB; CHARCHAR JM. 1997. Cultivo da cenoura (Daucus carota L). Brasília: EMBRAPA-CNPH, 15p. (Instruções Técnicas da Embrapa Hortaliças, 13). 